\title{
Auf dem Weg zur Ganztagsschule
}

\section{Das Jugendbegleiter-Programm in Baden-Württemberg}

STEFANIE WICHMANN

Stefanie Wichmann ist Mitarbeiterin der Jugendstiftung Baden-Württemberg. Sie ist Ansprechpartnerin für das Jugendbegleiter-Programm des Landes.

www.jugendbegleiter.de

\author{
Das Jugendbegleiter-Programm des Landes Baden- \\ Württemberg ermöglicht seit 2006 in umfassender \\ und vielfältiger Weise außerunterrichtliche \\ Bildungs- und Betreuungsangebote an öffentlichen \\ Schulen durch ehrenamtliche Kräfte und \\ außerschulische Partner. Mittlerweile nehmen rund
1.600 Schulen am Jugendbegleiter-Programm teil.
}

Das Konzept des Programms in Stichworten:

- Es unterstützt Schulen bei der Entwicklung eines auf die lokalen Begebenheiten und Bedürfnisse abgestimmten Bildungskonzepts.

- Abhängig von der Anzahl der angebotenen Wochenstunden im Rahmen des Programms erhalten Schulen Fördermittel, mit denen sie eigenverantwortlich Angebote realisieren können. Zusätzlich können Schulen ein Kooperationsbudget beantragen, wenn sie mit außerschulischen gemeinnützigen Partnern innerhalb des Jugendbegleiter-Programms kooperieren.

- Für Sachkosten sowie Fortbildungsund Koordinierungskosten können jeweils bis zu 20 Prozent des Grundbudgets abgerechnet werden.

n In jedem Schuljahr müssen mindestens vier Zeitstunden pro Woche durch Jugendbegleiterinnen und Jugendbegleiter angeboten werden, um eine Förderung zu erhalten.

- Jugendbegleiter-Angebote finden verlässlich jede Woche für mindestens ein Schulhalbjahr statt.

- Die Mindestgruppengröße beträgt fünf Schülerinnen und Schüler.

Aktuell realisieren mehr als 22.400 Jugendbegleiterinnen und Jugendbegleiter im Land ehrenamtlich über 43.200 Stunden Bildungs- und Betreuungsangebote pro Woche. Sie sind damit zu einem wichtigen Stützpfeiler in der
Schullandschaft Baden-Württembergs geworden. Die Anzahl der beteiligten Schulen hat sich somit in sieben Jahren mehr als versechsfacht.

Das Jugendbegleiter-Programm wird von der Jugendstiftung BadenWürttemberg im Auftrag des Ministeriums für Kultus, Jugend und Sport Baden-Württemberg durchgeführt. Eine Servicestelle berät die teilnehmenden Schulen und die außerschulischen Partner bezüglich eines Jugendbegleiter-Konzepts, gibt Informationen zu finanziellen und rechtlichen Fragen und stellt Arbeits- und Planungshilfen zur Umsetzung des JugendbegleiterProgramms an der betreffenden Schule und im lokalen Bildungsnetzwerk zur Verfügung.

\section{Wie das Projekt arbeitet}

Bildung, Betreuung und Erziehung sind nicht nur Aufgaben der Eltern und der Schule, sondern liegen in der Verantwortung der ganzen Gesellschaft. Eine Grundintention des Jugendbegleiter-Programms ist es deshalb, die Öffnung von Schulen für die Mitarbeit außerschulischer Partner und freiwillig Engagierter zu fördern. Das Jugendbegleiter-Programm bildet eine Brücke zwischen Lebensraum Schule, Menschen aus dem lokalen Umfeld und Vertreterinnen und Vertreter von Institutionen und Einrichtungen, die an der Gestaltung der Schule mitwirken wollen. 


\section{Zahlen und Fakten zum Jugendbegleiter-Programm in Baden-Württemberg}

- Aktuell engagieren sich 22.464 Jugendbegleiterinnen und Jugendbegleiter ehrenamtlich an 1.582 Schulen in BadenWürttemberg.

- Die ehrenamtlich Tätigen bieten 43.290 Zeitstunden pro Schulwoche im Rahmen des Jugendbegleiter-Programms an.

- 43 Prozent aller Jugendbegleiterinnen und Jugendbegleiter sind organisationsunabhängige Einzelpersonen, dar- unter Eltern, Studenten, Senioren u. v. a. 38 Prozent stammen aus der (älteren) Schülerschaft und 19 Prozent gehören einem Verein, Verband oder einer Organisation an.

- Insgesamt bewerten 94 Prozent aller Schulleitungen das JugendbegleiterProgramm mit "sehr positiv" oder "positiv«. Lediglich vier Schulen geben ein negatives Votum.
- Durchschnittlich 27 Wochenstunden Bildungs- und Betreuungsangebote werden an den Jugendbegleiter-Schulen in unterschiedlichen Themengebieten wie Medien, Kunst und Kultur, Wirtschaft, Technik, Sport sowie Hausaufgabenbetreuung oder Musik angeboten.
Die beteiligten Schulen erhalten - abhängig von der angebotenen Wochenstundenzahl - Fördermittel des Landes, die sie eigenverantwortlich nutzen und mit denen sie Angebote realisieren können. Zusätzlich haben die Schulen die Möglichkeit, ein Kooperationsbudget $\mathrm{zu}$ beantragen, wenn sie mit außerschulischen gemeinnützigen Organisationen innerhalb des JugendbegleiterProgramms kooperieren.

Mit dem zur Verfügung gestellten Förderbudget können die Schulen Aufwandsentschädigungen, Sachkosten sowie Fortbildungs- und Koordinierungs- und das Ehrenamt in den schulischen Lebensraum junger Menschen intensiv einbezogen. Der Zugang der Schülerinnen und Schüler zur außerschulischen Partnern, beispielsweise aus der Jugendarbeit oder dem Sport, wird bewusst hergestellt.

Lokale Bildungsnetzwerke schaffen Bildungsräume, in denen mit vielfältigen Bildungs- und Betreuungsangeboten gezielt auf die Bedürfnisse, Stärken und Fähigkeiten der Schülerinnen und Schüler eingegangen werden kann. Die Internet-Plattform »Lokale Bildungsnetzwerke Baden-Württemberg « bietet

\section{"Bildung, Betreuung und Erziehung sind letztlich Aufgaben der ganzen Gesellschaft»}

kosten bestreiten. Voraussetzung ist die Kontinuität der Jugendbegleiter-Angebote an den Schulen: diese finden verlässlich mindestens ein Schulhalbjahr lang statt und umfassen mindestens vier Zeitstunden in der Woche.

\section{Lokale Bildungsnetzwerke bieten Möglichkeiten für Kooperationen}

Mit dem Jugendbegleiter-Programm können Schulen ihr individuelles Profil entwickeln und stärken. Verschiedene Lernorte werden miteinander verbunden, so dass lokale Bildungsnetzwerke entstehen. Dabei lernen Kinder und Jugendliche auch Jugendbegleiterinnen und Jugendbegleiter aus unterschiedlichen Tätigkeitsfeldern kennen. Gleichzeitig wird die gemeinsame Arbeit aller Personen, die für Kinder und Jugendliche Verantwortung tragen, gestärkt
Schulen, außerschulischen Partnern und Interessierten unter www.bildungsnetzwerke-bw.de verschiedene Recherche-, Präsentations- und Kommunikationsmöglichkeiten.

Schulen erhalten somit die Möglichkeit, die Visualisierung ihrer Lokalen Bildungsnetzwerke selbstständig im Netz aufzubauen und zu pflegen. Die Lokalen Bildungsnetzwerke der einzelnen Schulen lassen sich über die Suchfunktion der Baden-WürttembergLandkarte oder über die gezielte Stadtsuche finden. Informationen zu den verschiedenen Kooperationspartnern und der Schule sind abrufbar. Weiterhin besteht die Möglichkeit, gezielt nach Schulen zu suchen, die an weiteren Kooperationen interessiert oder auf der Suche nach geeigneten Jugendbegleiterinnen und Jugendbegleitern sind.

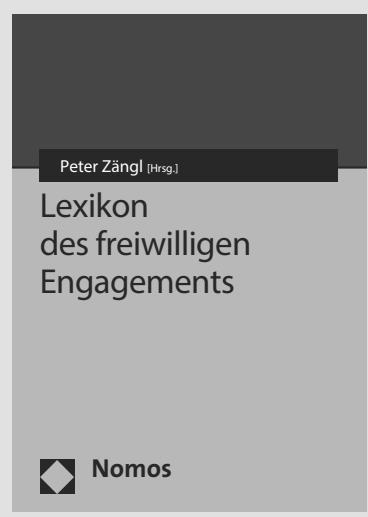

Lexikon des freiwilligen Engagements

Herausgegeben von Prof. Dr. Peter Zängl

2014, ca. 500 S., brosch., ca. 49,- ISBN 978-3-8329-7248-6

Erscheint ca. März 2014

Dieses Lexikon systematisiert und definiert rund 300 Schlüsselbegriffe, erläutert diese anhand von praktischen Beispielen und ist Wegweiser für weitere Recherchen.

Das Werk wendet sich an NonProfit und For-Profit-Organisationen, Verbände und Vereine, Bildungsträger, Gebietskörperschaften sowie freiwillig Engagierte und ehrenamtlich Tätige.

www.nomos-shop.de/14401

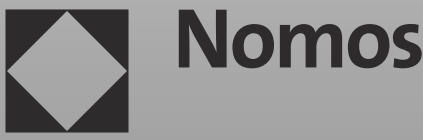

